\title{
Epidemiologie und Nosologie der Anisakiose, einer in Mitteleuropa selten diagnostizierten Helminthozoonose
}

\section{An den Herausgeber}

Ich möchte den Autoren des Manuskripts „Epidemiologie und Nosologie der Anisakiose, einer in Mitteleuropa selten diagnostizierten Helminthozoonose - zwei Fallberichte" gratulieren für die sehr interessante und pekuliäre klinische, multidisziplinäre Aufarbeitung der zwei beschriebenen Fallberichte [1]. Insbesondere wurde sehr interessant dargestellt, wie Reste von Anisakis simplex mittels molekularbiologischen Methoden in einem resezierten Ileumabschnitt detektiert werden konnte.

In Spanien diagnostizieren und behandeln wir seit einem Jahrzehnt ein in diesem Lande sehr prävalentes Krankheitsbild, das als gastro-allergische Anisakiasis beschrieben wurde und mit allergischen akuten Symptomen (Urtikaria, Quinke-Ödem oder Anaphylaxis) einhergeht und der akuten gastrischen Parasitierung durch Anisakis simplex parallelgeht $[2,3]$. Es wurde vermutet, dass die Präsenz von allergischen Erscheinungen einen Mechanismus darstellt, die Larven aus der Magen-Mukosa zu expulsieren, damit es nicht zu chronischen Erscheinungen wie die dargestellten kommt.

Obwohl dieses Krankheitsbild durch die akuten Symptome nach Fischgenuss anamnestisch leichter zu diagnostiziern ist, möchte ich dennoch auf diagnostische Möglichkeiten hinweisen, die in Fällen, wie den von Auer et al. beschriebenen, auch von Nutzen sein kann.

So wissen wir heute, dass jede Parasitierung, mit oder ohne allergische Begleiterscheinungen, von einer polyklonalen Stimulierung aller Immunoglobulin-Isotypen begleitet wird, die diagnostisch von Nutzen sein können. Es ist wahrscheinlich, dass der Nachweis von spezifischer IgE gegen Anisakis simplex weniger Kreuzreaktivität aufweist, dies ist mittlerweile mit kommerziell erhältlichen Systemen möglich, z.B. mittels CAP-FEIA von Phadia Diagnostics. Andererseits ist es auch möglich, das Vorhandensein von spezifischer IgE mittels Hauttestung mit ebenfalls erhältlichen Extrakten zu diagnostizieren. In Gebieten, in denen die Prävalenz solcher Antikörper als sehr selten zu erwarten ist, wäre eine solche Testung von fast pathognomonischer Bedeutung. Darüber hinaus ist es möglich, dem Vorhandensein solcher Antikörper eine positive diagnostische Bedeutung zuzuordnen, wenn nach mehreren Monaten, nach anfänglichem Steigen, ähnlich wie bei fast allen Infektionserkrankungen, eine signifikante Abnahme zu verzeichnen ist. Da in den zwei beschriebenen Fällen IgGAntikörper nachgewiesen wurden, wäre eine deutliche Abnahme des Titers nach mehreren Monaten ebenfalls von diagnostischer Bedeutung, um auszuschließen, dass diese von möglichen früheren Kontakten herstammen [4].

\section{Literatur}

1. Auer H, Leskowschek H, Engler J, Leitner G, Wentzel C, Wolkerstorfer W (2007) Epidemiologie und Nosologie der Anisakiose, einer in Mitteleuropa selten diagnostizierten Helminthozoonose- zwei Fallberichte. Wien Klin Wochenschr 119 [Suppl 3]: 106-109

2. Daschner A, Alonso-Gómez A, Cabañas R, Suarez-deParga JM, López-Serrano MC (2000) Gastro-allergic Anisakiasis: borderline between food allergy and parasitic disease - Clinical and allergological evaluation of 20 patients with confirmed acute parasitism by Anisakis simplex. J Allergy Clin Immunol 105: 176-181

3. Alonso A, Daschner A, Moreno-Ancillo A (1997) Anaphylaxis with Anisakis simplex in the gastric mucosa. NEJM 337: 351-352

4. Daschner A, Alonso-Gómez A, Caballero MT,Suarez-deParga JM, López-Serrano MC (1999) Usefulness of early serial measurement of specific and total $\operatorname{IgE}$ in the diagnosis of Gastro-allergic Anisakiasis. Clin Exp Allergy 29: $1260-1264$

Korrespondenz: Dr. A. Daschner, Servicio de Alergia, Hospital Universitario La Princesa, C/Diego de León 62, 28006 Madrid, Spanien, E-mail: adaschmer@meditex.es

\section{Antwort der Autoren}

Eineinhalb Jahre nach der Registrierung und Dokumentation der ersten zwei Anisakiose-Fälle in Österreich sind wir gerade dabei, einen weiteren Fall eines möglichen bis sehr wahrscheinlichen Anisakis-Befalls diagnostisch abzuklären. Wir nützen dabei den vor kurzem in unserem Labor etablierten Anisakis-Westernblot sowie molekularbiologische Methoden zur Speziesbestimmung. Als rein parasitologische Institution ohne Allergielaboratorium ist es uns derzeit (noch) nicht möglich mittels CAP-FEIA eine gastroallergische Anisakiose zu diagnostizieren bzw. auszuschließen. Wir werden jedoch in Zukunft die labordiagnostische Abklärung einer gastroallergischen Anisakiose differentialdiagnostisch im Auge behalten und uns gegebenenfalls mit Allergielabors, die ihre Patienten mittels CAP-FEIA „screenen“, zusammenschließen. Wir sechs Autoren und Koautoren, die wir die ersten Anisakiose-Fälle in Österreich beschrieben haben, freuen uns jedenfalls ob des groBen fachlichen Kompliments, das wir vom Allergologen und Anisakis-Experten Dr. Alvaro Daschner vom Hospital Universitario La Princes, Madrid, Spanien bekommen haben.

Herbert Auer im Namen aller Koautoren

Korrespondenz: Univ.-Prof. Dr. Herbert Auer, Klinisches Institut für Hygiene und Medizinische Mikrobiologie, Kinderspitalgasse 15, 1090 Wien, E-mail: herbert.auer@meduniwien.ac.at 